\title{
Dietary pattern longitudinality during 8 years in children: results from the European Longitudinal Study of Pregnancy and Childhood (ELSPAC-CZ)
}

\author{
Markéta Grulichová ${ }^{1, *}$, Filip Zlámal ${ }^{1,2}$, Lenka Andrýsková2 ${ }^{2}$ Jan Švancara², \\ Hynek Pikhart ${ }^{2,3}$ and Julie Bienertová-Vaškủ ${ }^{1,2}$ \\ 'Department of Pathological Physiology, Faculty of Medicine, Masaryk University, Brno 625 00, Czech Republic: \\ ${ }^{2}$ Research Centre for Toxic Compounds in the Environment (RECETOX), Masaryk University, Brno, Czech Republic: \\ ${ }^{3}$ Research Department of Epidemiology and Public Health, University College London, London, UK
}

Submitted 15 April 2019: Final revision received 29 February 2020: Accepted 19 March 2020: First published online 25 June 2020

\begin{abstract}
Objective: Dietary pattern analysis constitutes a suitable method for identifying complex food preferences as well as a useful tool for comparing dietary behaviour across individual populations. In addition to a lack of information on Central European dietary patterns, dietary data featuring a longitudinal aspect are likewise largely unavailable for the region. Our study thus strives to address this gap by analysing children's dietary patterns, their stability and possible changes at 7 , 11 and 15 years in the Czech part of the European Longitudinal Study of Pregnancy and Childhood (ELSPAC-CZ).

Design: We analysed dietary data based on the self-reported semi-quantitative FFQ obtained in 1998, 2002 and 2006. Dietary patterns were derived using factor analysis for each period, followed by the determination of dietary pattern stability across the individual periods.

Setting: The analysis of dietary patterns was based on longitudinal children's dietary data from the geographical region that was undergoing massive socio-economic changes at the time of birth of the study subjects.

Participants: All participants were children. At 7 years the analysis included 3220 children, at 11 years the analysis included 2509 children and at 15 years the analysis included 1589 children.

Results: Two stable children's dietary patterns labelled as 'prudent' and 'junk food' were identified across all three time points (7, 11 and 15 years).

Conclusions: This study identifies stable longitudinal trends in the dietary behaviour of children enrolled in the ELSPAC-CZ study.
\end{abstract}

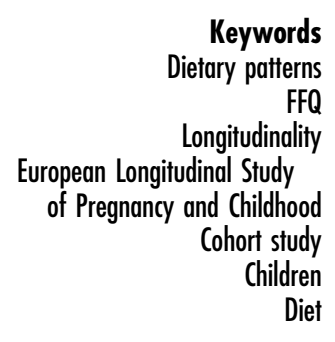

Poor diet in early life is a major modifiable risk factor with a multitude of health outcomes. Although numerous studies have focused on the association between defined health outcomes and diet, many of these studies are frequently plagued by methodological discrepancies and often feature a cross-sectional design which represents a limitation when attempting to interpret long-term trends ${ }^{(1)}$. The geographical location of a given country is also an important factor influencing the quality of data, as in some areas data collection had been hindered due to political reasons for a long time. With some exceptions, such as the HAPIEE study (Health, Alcohol and Psychosocial factors In Eastern Europe), which focused primarily on the elderly ${ }^{(2)}$, this long-term lack of quality follow-up on the dietary characteristics of country-wide populations as well as specific subpopulations also applies to Central Europe. Even though a recent large-scale study performed on data from nineteen countries focused on clustering energy balancerelated behaviours and on associating identified clusters with weight status using data on the fruit and vegetable intake of Eastern European children and adolescents ${ }^{(3)}$, complex evaluation of the diet and especially its longitudinal outcomes in children is largely missing ${ }^{(4-8)}$.

Some information on the dietary behaviour of Central European children comes from a Czech study entitled Health Behaviour in School-aged Children. While the 
Health Behaviour in School-aged Children study focused on the consumption of fruit, vegetables, soft drinks and sweets by Czech children and adolescents from 2002 to $2014^{(9)}$, it did not focus specifically on dietary patterns. Recently, the dietary patterns of pregnant women enrolled in the European Longitudinal Study of Pregnancy and Childhood (ELSPAC-CZ) study were analysed by Bienertová-Vašků et al. ${ }^{(10)}$. However, longitudinal studies describing the dietary patterns of children are currently missing. Moreover, this lack of data must also be viewed in the context of major socio-economic and political changes which took place in Central Europe beginning with the early 1990s: these extensive and profound changes may be expected to have resulted in substantial dietary behaviour changes within the space of the past 20 years.

In the 1990s, Czech society underwent a unique transition from a planned economy to a market-based system; this historical occurrence may be viewed as a suitable basis for studying the impact of socio-economical changes on the dietary behaviour of entire families ${ }^{(11)}$. Like most Central and Eastern European countries, Czechoslovakia (subsequently split into the Czech Republic and Slovakia) experienced a transitional recession period in the early 1990 s as a result of an inflation rate jump. While unemployment rose steeply in Slovakia during the recession of 1991-1992 (14.7\% in 1993), it remained stable in the Czech Republic ( $4 \%$ in 1993). Although the Czech Republic and Slovakia experienced a rise in income inequality during the transition period $^{(11)}$, secondgeneration reform policies adopted by the Czech government to correct the shortcomings of first-generation reforms subsequently led to sustained economic growth beginning with the mid-1990s.

In order to address the issue of dietary pattern stability in a profoundly changing population undergoing a major socio-economic transformation, this study aims to investigate the longitudinal aspects of dietary behaviour - assessed in the form of dietary patterns - between 1991 and 2006 in the ELSPAC-CZ. This study specifically aims to investigate: (i) the dietary patterns of children at 7, 11 and 15 years of age, (ii) the stability (level of adherence) of the observed patterns throughout a follow-up period of 8 years and (iii) the predisposing factors for identified dietary patterns in the observed population.

\section{Methods}

\section{Study design}

The ELSPAC is a population-based study examining the effects of biological, psychosocial, economic and environmental factors; the study focuses on how these factors affect the health and development of children from the prenatal period to adulthood. The study was initiated by the World Health Organization Regional Office for Europe in
1985 with the aim of collecting data from 40000 children across Europe ${ }^{(12)}$. The project was originally coordinated by Bristol University - Avon Longitudinal Study of Pregnancy and Childhood (ALSPAC) ${ }^{(13)}$ - and operated by eight independent centres based in the UK, Czechoslovakia, Greece, Ukraine and Russia (the latter two were initially part of the USSR). Protocol development, including follow-up planning and questionnaire design, was coordinated by ALSPAC ${ }^{(14)}$. The Czech portion of the study (ELSPAC-CZ) included eligible mothers from the Brno (the second largest city in the Czech Republic) that was home to 389000 inhabitants in 1991-1992 ${ }^{(15)}$. All participating mothers were expected to deliver between 1 April 1991 and 30 June 1992. Selfreported questionnaires filled out by participants included records of demographics, lifestyle, dietary habits, partnership, life attitudes, life events, social factors and environmental exposure. In addition to its primary aims, the Czech ELSPAC-CZ study also examined socio-economic changes related to the societal transformation after the fall of Communism in $1989^{(10,14)}$.

\section{Exclusion criteria and final sample size}

The analysis presented in this study is based on data collected from the mothers in the study who reported on their children at two time points: 7 and 11 years. The children themselves reported on their dietary behaviour at 15 years of age. For ease of reporting, we will refer to these time points as 7, 11 and 15 years throughout the paper. The cases (children of reporting mothers or the reporting children themselves at 15 years) were excluded from the analysis in case the absolute values of their factor scores exceeded 3.5. A total of $0.9 \%$ of all subjects were excluded from the study as a result of extreme factor scores. At 7 years, the analysis thus included 3230 children, at 11 years the analysis included 2509 children and at 15 years the analysis included 1589 children.

\section{Dietary assessment}

Dietary data were obtained from a self-reported semiquantitative FFQ (SFFQ) collected according to the ELSPAC-CZ protocol from all study participants. The SFFQ was based on ALSPAC SFFQ, with several local food items added. Data were collected from children at three time points. Mothers were asked to fill in SFFQ about each child's diet around the seventh and eleventh birthday of their child. The children were asked to fill out the SFFQ themselves at 15 years of age.

Participants were asked to indicate consumption frequency for 40-60 food questions using the following criteria: (1) never or rarely; (2) twice a month; (3) one to three times per week; (4) four to seven times per week and (5) several times a day. Participants were also asked to report portion sizes. For factor analysis purposes, a score for each component was created by multiplying 
the factor loadings by a standardised value for each food item and summing through all food items. All food items were divided into thirty-nine food groups. Food groups with no detectable effects were removed for greater clarity. This method has been used before, for example, by the HAPIEE study ${ }^{(2,10)}$. A detailed description of food item grouping across investigated time periods is provided in Supplemental Table 1.

\section{Statistical analysis}

Children's dietary patterns were estimated by exploratory factor analysis with varimax rotation for thirty-nine food groups. The Kaiser-Olkin-Meyer test was used to identify whether factor analysis results were appropriate. When a suitable number of factors were identified for a given group and period, factor scores were extracted. To determine the longitudinal stability of individual dietary patterns, factor analysis was performed again, this time with extracted factor scores from the previous analysis. The Pearson correlation coefficient was used to assess the association between extracted factor scores for a given pair of dietary patterns across observed time periods. This method was selected due to the high dropout rate of subjects when considering analyses of full cases from cross-sectional and longitudinal point of view. Food groups with absolute factor loadings greater than $0 \cdot 20$ for a given dietary pattern were considered to significantly contribute to the pattern, and conversely, negative loadings lower than -0.2 were considered to contribute inversely to the dietary pattern. All analyses were performed using $\mathrm{R}$ software, version 3.3.3. $P$-values of less than 0.05 were considered statistically significant.

\section{Results}

\section{Descriptive results}

Description of the biological characteristics of the children in the study is given in Table 1 . The percentage of total variance the dietary patterns account for stood at $14.8 \%$ at 7 years, $20.4 \%$ at 11 years and $25.5 \%$ at 15 years.

\section{Children's dietary patterns}

Three dietary patterns were identified at the three examined time points $(7,11$ and 15 years). The pattern scores at each time point are provided in Table 2. Consumption at 7 years was characterised by two dietary patterns, labelled 'prudent' and 'junk food'. Consumption at 11 years was characterised by three dietary patterns, labelled 'junk food', 'prudent 1' and 'prudent 2'. Consumption at 15 years was characterised by two dietary patterns, labelled 'junk food' and 'prudent'. Although three separate patterns were thus identified, two dietary patterns were found to be consistently present in children across all three time points: the 'junk food' and the 'prudent' pattern. The trajectories of children's dietary patterns across each time period and between individual time periods

Table 1 Non-dietary characteristics of children

\begin{tabular}{|c|c|c|c|c|c|c|c|c|c|c|c|c|}
\hline \multirow[b]{3}{*}{ Age } & \multicolumn{4}{|c|}{7 years } & \multicolumn{4}{|c|}{11 years } & \multicolumn{4}{|c|}{15 years } \\
\hline & \multicolumn{2}{|c|}{ Girls } & \multicolumn{2}{|c|}{ Boys } & \multicolumn{2}{|c|}{ Girls } & \multicolumn{2}{|c|}{ Boys } & \multicolumn{2}{|c|}{ Girls } & \multicolumn{2}{|c|}{ Boys } \\
\hline & Mean & SD & Mean & SD & Mean & SD & Mean & SD & Mean & SD & Mean & SD \\
\hline$n$ & 1371 & & 1445 & & 1048 & & 1040 & & 716 & & 687 & \\
\hline Age (years) & $7 \cdot 0$ & 0.1 & $7 \cdot 0$ & 0.1 & 11.0 & 0.1 & 11.0 & 0.1 & $15 \cdot 0$ & 0.1 & $15 \cdot 0$ & $0 \cdot 1$ \\
\hline Birth weight/weight (kg) & $24 \cdot 1$ & $4 \cdot 1$ & 25.0 & 4.2 & $39 \cdot 3$ & 8.5 & $39 \cdot 3$ & $8 \cdot 3$ & 56.5 & $8 \cdot 7$ & $62 \cdot 1$ & $11 \cdot 7$ \\
\hline Birth length/height $(\mathrm{cm})$ & 124.5 & $5 \cdot 8$ & 125.5 & 5.4 & $148 \cdot 7$ & 7.4 & $148 \cdot 1$ & $6 \cdot 6$ & 165.9 & 6.5 & $174 \cdot 6$ & 7.4 \\
\hline $\mathrm{BMI}\left(\mathrm{kg} / \mathrm{m}^{2}\right)$ & $15 \cdot 5$ & 1.9 & $15 \cdot 8$ & $2 \cdot 0$ & $17 \cdot 7$ & 2.9 & $17 \cdot 8$ & 2.9 & 20.5 & 2.9 & $20 \cdot 3$ & $3 \cdot 1$ \\
\hline Prudent pattern 1 & 0.02 & 0.96 & -0.02 & 1.04 & 0.03 & 0.96 & -0.01 & 1.05 & 0.00 & 0.98 & 0.01 & 1.00 \\
\hline Prudent pattern 2 & - & & - & & 0.03 & 1.00 & -0.04 & 0.97 & - & & - & \\
\hline Junk food & -0.03 & 0.98 & 0.02 & 1.01 & -0.04 & 1.01 & 0.02 & 0.97 & -0.24 & $0.98^{*}$ & 0.24 & $0.96^{*}$ \\
\hline \multicolumn{13}{|l|}{ Height $z$-score $(-)$} \\
\hline Mean & 0.65 & & & & 0.62 & & & & 0.66 & & & \\
\hline SD & 1.01 & & & & 1.04 & & & & 0.94 & & & \\
\hline \multicolumn{13}{|l|}{ BMI $z$-score $(-)$} \\
\hline Mean & -0.01 & & & & 0.06 & & & & -0.02 & & & \\
\hline \multirow[t]{2}{*}{ SD } & 1.11 & & & & 1.18 & & & & 1.00 & & & \\
\hline & & & & & $n$ & $\%$ & $n$ & $\%$ & $n$ & $\%$ & $n$ & $\%$ \\
\hline $\begin{array}{l}\text { Maternal education: } \\
\text { completed primary } \\
\text { school }\end{array}$ & \multicolumn{4}{|c|}{ Unknown } & 262 & $26 \cdot 4$ & 282 & $28 \cdot 4$ & 133 & 19.2 & 142 & 21.2 \\
\hline $\begin{array}{l}\text { Maternal education: } \\
\text { completed secondary } \\
\text { school }\end{array}$ & \multicolumn{4}{|c|}{ Unknown } & 456 & $45 \cdot 9$ & 434 & 43.8 & 343 & 49.6 & 313 & $46 \cdot 8$ \\
\hline $\begin{array}{l}\text { Maternal education: } \\
\text { completed university }\end{array}$ & \multicolumn{4}{|c|}{ Unknown } & 276 & $27 \cdot 8$ & 276 & $27 \cdot 8$ & 216 & $31 \cdot 2$ & 214 & $32 \cdot 0$ \\
\hline
\end{tabular}

Height $z$-score, height-for-age $z$-score; BMI $z$-score, BMl for-age $z$-score.

*Statistically significant difference, $P<0.001$. 
Table 2 Factor loading scores for relationships between food groups and children's dietary patterns*

Children's dietary patterns

\begin{tabular}{|c|c|c|c|c|c|c|c|}
\hline \multirow{2}{*}{$\frac{\text { Age }}{\text { Food groups }}$} & \multicolumn{2}{|c|}{7 years } & \multicolumn{3}{|c|}{11 years } & \multicolumn{2}{|c|}{15 years } \\
\hline & JF & PP & JF & PP1 & PP2 & JF & PP \\
\hline White bread & & & & & & 0.22 & \\
\hline Whole-wheat bakery products & & 0.40 & & & & & \\
\hline Breakfast cereals & & 0.38 & & & 0.29 & & 0.45 \\
\hline Biscuits & 0.51 & & 0.58 & & & 0.52 & \\
\hline Yoghurts, puddings & 0.20 & 0.45 & & & 0.69 & 0.28 & 0.45 \\
\hline Pies and cakes & 0.24 & & 0.45 & & 0.22 & 0.41 & 0.23 \\
\hline Poultry & & 0.35 & & 0.34 & & $0 \cdot 27$ & 0.25 \\
\hline Red meat & & & & 0.31 & & 0.29 & 0.20 \\
\hline Pâtés/ground meat & 0.35 & & & & & 0.63 & \\
\hline Offal, giblets & & & & 0.24 & & 0.32 & 0.30 \\
\hline Sausages & 0.36 & & 0.23 & & & 0.44 & \\
\hline Pizza & 0.31 & & & & & 0.58 & \\
\hline Fish & & 0.42 & & 0.39 & 0.22 & & 0.47 \\
\hline Molluscs & 0.20 & & & & & & \\
\hline Eggs & & 0.23 & & 0.28 & & 0.31 & 0.34 \\
\hline Cheese & & 0.33 & & & 0.63 & & 0.32 \\
\hline Legumes & & 0.43 & & 0.43 & & & 0.52 \\
\hline Nut and seeds & 0.25 & 0.25 & 0.33 & 0.23 & & 0.35 & 0.41 \\
\hline French fries & 0.53 & & & & & 0.68 & \\
\hline Boiled potatoes & & 0.27 & & 0.26 & & & 0.32 \\
\hline Dumplings & 0.23 & & & 0.37 & & 0.30 & 0.34 \\
\hline Pasta & & 0.39 & & 0.55 & & & 0.45 \\
\hline Rice & & 0.40 & & 0.56 & & & 0.43 \\
\hline Vegetables & & 0.59 & & 0.36 & 0.46 & & 0.62 \\
\hline Root vegetables & & 0.51 & & & & & \\
\hline Fresh fruit & & 0.43 & & & 0.53 & & 0.56 \\
\hline Fresh juice & & 0.31 & 0.21 & & & 0.32 & 0.42 \\
\hline Cola & 0.47 & & 0.34 & & & 0.63 & \\
\hline Tea & & & & & & & 0.31 \\
\hline Herbal tea & & 0.20 & & & & & 0.39 \\
\hline Sweeteners & 0.25 & & & & & & \\
\hline Chocolate & 0.48 & & 0.62 & & & 0.65 & \\
\hline Sweetmeats & 0.36 & & 0.61 & & & 0.60 & \\
\hline Ice-cream & & 0.39 & & & & 0.57 & \\
\hline Ketchup, mustard & 0.41 & & 0.40 & & & & \\
\hline Milk & & 0.31 & & & 0.38 & & 0.31 \\
\hline Soft drinks & 0.41 & & 0.40 & & & 0.50 & \\
\hline Salty snacks & 0.48 & & 0.56 & & & 0.68 & \\
\hline Desserts & & & 0.48 & & & 0.59 & 0.21 \\
\hline
\end{tabular}

PP, prudent pattern; JF, junk food; PP1, prudent pattern 1; PP2, prudent pattern 2.

${ }^{*}$ Factor loadings with absolute value $<0.2$ not shown.

are provided in Fig. 1. A description of the full cases represented by all 1364 children with dietary patterns available across all three time points is provided in Fig. 2 .

An analysis of correlations across investigated time points showed the consistent presence of the 'prudent' pattern across all three time points. The 'junk food' dietary pattern was consistently characterised by typical high-fat and high-sugar foods. The correlation obtained using the Pearson correlation coefficient $(0.85)$ showed the robust presence of the 'junk food' dietary pattern across investigated time points.

Furthermore, the calculated factor scores were evaluated using the univariate regression models in relation to socioeconomic and maternal biological factors (Supplemental Table 2). Maternal age at birth, maternal education, maternal BMI before pregnancy, substance abuse, alcohol consumption, smoking and relationship status were analysed.
Although no significant associations were observed for the first two time points in the study, some of the evaluated variables were significantly correlated with the presence of the 'junk food' pattern at 15 years: maternal education (6.82\%), maternal age at birth (6.24\%), maternal BMI before pregnancy (6.08\%), substance abuse ( $5.31 \%)$, maternal alcohol intake during pregnancy (5.48\%), maternal smoking during pregnancy (5.80\%) and maternal relationship status $\left(5.05 \%\right.$ ) (multiple $R^{2}$ values in the linear regression model for the variable determining the factor score in dependency on gender, evaluated variable and their interaction).

We also investigated the relationship between the dropout of children between investigated time points and other above-mentioned variables. Dropout between 7 years and 11 years was significantly associated with maternal age (non-missings were older than missings by approximately 
Dietary patterns in Czech children

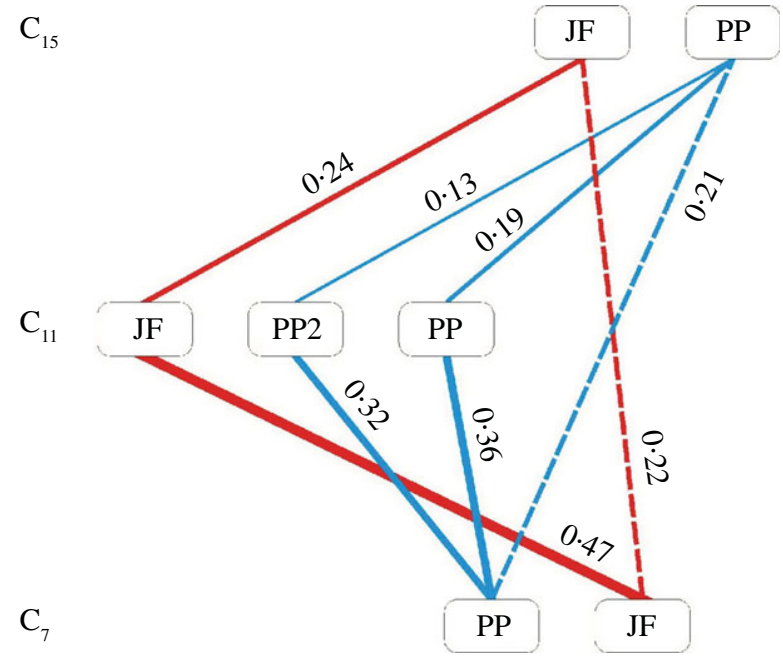

Fig. 1 (colour online) Stability and changes in children's dietary patterns across three time periods. The reported values are the Pearson pairwise correlation coefficient for a pair of factor scores corresponding to given dietary patterns across the investigated time points. Only statistically significant correlations are shown. PP1, prudent pattern 1; JF, junk food; PP2, prudent pattern $2 ; c_{7}$, children's 7 years time period; $c_{11}$, children's 11 years time period; $c_{15}$, children's 15 years time period. Line thickness is directly proportional to the correlation coefficient value. Solid lines connect two adjoining time periods (7-11 and 11-15). Dashed lines connect other time periods $(7-15)$

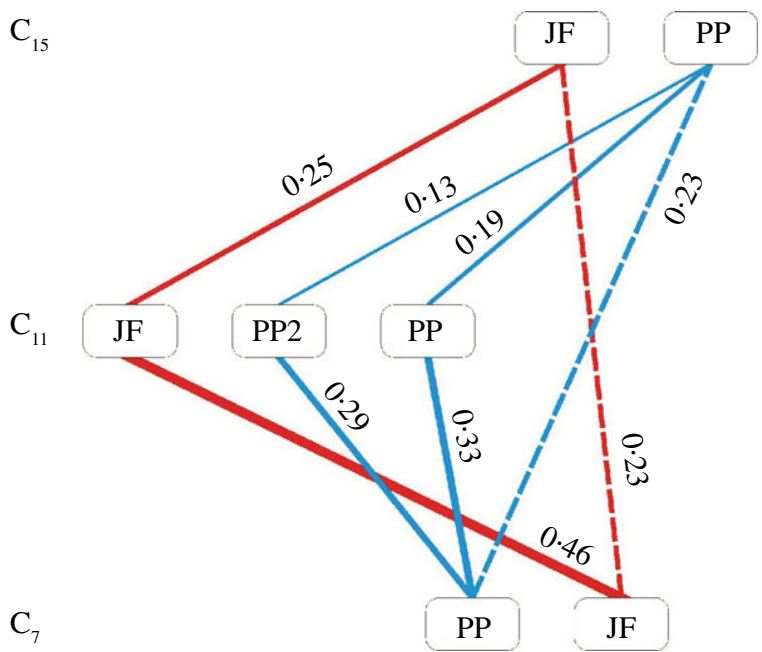

Fig. 2 (colour online) Stability and changes of children's dietary patterns from the total of 1364 children across all three time points. The reported values are the Pearson correlation coefficients for a pair of factor scores in full case analysis (where DP is known in all three ages) corresponding to given dietary patterns. Only statistically significant correlations are shown. PP1, prudent pattern 1; JF, junk food; PP2, prudent pattern 2; $\mathrm{C}_{7}$, children's 7 years time period; $\mathrm{c}_{11}$, children's 11 years time period; $c_{15}$, children's 15 years time period. Line thickness is directly proportional to the correlation coefficient value. Solid lines connect two adjoining time periods (7-11 and 11-15). Dashed lines connect other time periods (7-15)
0.4 years), 'junk food' pattern score at 7 years (missings had higher scores than non-missings), sex of the child (nonmissings: men and women were approximately equally likely to continue, missings: men were more likely to drop out, $\mathrm{OR}=1.23$ ) and maternal education (children of parents with higher education were more likely to continue in the study). Dropout between 11 and 15 years was significantly associated with maternal age (non-missings were older than missings by approximately 0.9 years), maternal height (non-missings were approximately $0.7 \mathrm{~cm}$ higher than missings), maternal BMI before pregnancy (missings had higher BMI than non-missings by approximately $0.3 \mathrm{~kg} / \mathrm{m}^{2}$ ), 'junk food' pattern score at 11 years (missings had higher scores than non-missings), sex of the child (women were more likely to be non-missings, whereas men were more likely to be missings, $\mathrm{OR}=1 \cdot 18$ ), maternal prenatal smoking status (more never smokers were found in the non-missing group) and maternal and paternal education (children of parents with higher education level were more likely to continue in the study).

\section{Discussion}

This study is the first to identify stable long-term trends in the dietary behaviour of children in a Central European longitudinal cohort in times of socio-economic transition. This study utilises data from the ELSPAC-CZ cohort, collected in 1998, 2002 and 2006, to analyse children's dietary patterns as well as their stability and changes at ages 7, 11 and 15 years. We analysed dietary data obtained using selfreported SFFQ and derived dietary patterns using factor analysis. We identified two stable dietary patterns in our study population of children (adolescents) which corresponded to patterns established in an ALSPAC population using the same methodology.

Study limitations include a significant dropout number among study subjects which may have biased the remaining data (discussed in detail in the Results). A second major limitation concerns the possible differences between data reported by proxies (mothers) at ages 7 and 11 and data reported by the study participants themselves at age 15 . A third limitation is posed by the distribution of SFFQ, submitted once per time point, and thus potentially incapable of recording all seasonal variations in food consumption. The last limitation of the study, that is, the unstable setting of a country transitioning from a planned economy to a market-based system, is also its greatest strength. It could be expected that a transition associated with rising social inequality and liquidity should have a profound effect on both nutritional composition and eating behaviours. However, it seems that within the observed period of almost two decades after the Velvet Revolution, the composition of children's diets remained relatively very stable, corresponding to patterns observed in the UK using the same methodology.

We observed two main stable patterns - a healthconscious one, labelled as 'prudent', and a 'junk food' 
pattern - which was consistently present across all three time points. While the 'junk food' pattern was consistently associated with higher consumption of biscuits, pies and cakes, sausages, cola and other soft drinks, and salty snacks, the 'prudent' pattern was associated with the consumption of breakfast cereals, yoghurts, fish, cheese, vegetables and fresh fruit and milk. Except for the absence of other types of meat than the red meat, the 'prudent' pattern is consistent with food pyramid-based recommendations valid at that time. It could be speculated that as the range of food items gradually expanded and the availability of various snacking options increased, pronounced changes in dietary behaviour of the study subjects would take place as a result of increased marketing pressure. However, no such occurrence was observed in this study, with observed dietary patterns remaining quite stable. It may thus be speculated that family dietary patterns have a more pronounced influence on children's diets than food item availability or massive marketing. However, it could also be suggested that the specific dietary of the family was due to other confounding variables.

In view of the above-mentioned profound socioeconomic transformation, it is rather surprising that the results of this study bear a striking similarity to outcomes reported in studies focusing on ALSPAC ${ }^{(5)}$. The results of such comparisons are even more compelling taking into account that the ELSPAC study generally used the same methodology in terms of the wording of the questionnaires as well as the frequency of administration to both the probands of the study and their proxies. Existing ALSPAC studies ${ }^{(4,5,16-18)}$ reported very similar dietary pattern ranges, which further supports the observation that patterns observed in the UK and in the Czech Republic were quite conserved. The greatest degree of similarity between ELSPAC and ALSPAC was observed between children's dietary patterns labelled 'prudent', that is, 'healthy' ${ }^{\prime 5,17)}$, 'health-conscious ${ }^{(4)}$, 'health aware'(16) and 'traditional/health-conscious ${ }^{\text {(4) }}$ and between children's dietary patterns labelled 'junk food' in this study, that is, 'processed $^{(4,5,17)}$. Taking into account the entirely different socio-economic environments of early 1990s UK and Czechoslovakia (and subsequently the Czech Republic), we may conclude that dietary patterns are relatively less susceptible to socio-economic trends and are probably much more conserved than originally expected. However, the reasons behind this are likely highly complex; a deeper understanding of the nature of this phenomenon thus requires further investigation.

Socio-economic transition generally indicates changes at the macro level and is expected to be a result of globalisation, urbanisation and industrialisation. It is expected that better improved economic situation status allows individuals to learn and live in safer and more friendly environments while achieving higher education levels ${ }^{(19)}$. Socio-economic changes are also inextricably linked to nutrition transition; however, this process has not been sufficiently documented. In a recent study which empirically examined the system of transitions using data from three countries (Indonesia, South Korea and the USA) at different stages of development, only vegetable oil consumption was found to have significantly increased in all three nations, with no particular significant trends established with respect to nutrition transition ${ }^{(20)}$. However, this publication assessed nutrition transition using either a percentage of energy obtained from carbohydrates, protein and fat ${ }^{(19)}$ or based on a few items selected from FFQ: rice, sugar and sweeteners, vegetable oil, animal fat, milk and meat ${ }^{(20)}$. A study focusing on a society in transition was conducted in South Africa, evaluating the nutritional records of 5-year-old urban black South African children from 1984 to $1995^{(21)}$. The study revealed a robust shift towards a more 'western' diet: while the 1984 diet consisted of $30 \%$ fat and $61 \%$ carbohydrates, fat intake had increased to $41 \%$ of total energy, while carbohydrate intake had decreased to $52 \%$ in 1995 . However, FFQ were only used for the evaluation of 1995 records, and the overall results are thus difficult to interpret.

In general, the effects of socio-economic transitions on nutritional determinants are vast, complex and far from adequately understood. No unifying trend capable of explaining the effects of socio-economic circumstances on food item choices and overall eating behaviour of populations or subpopulations has thus far been identified.

When analysing the influence of selected variables on dietary pattern distribution, we observed a strong influence of some investigated variables (maternal age, maternal education, substance abuse, maternal alcohol intake during pregnancy and maternal smoking during pregnancy) on pattern distribution, with the influence of maternal education and smoking most pronounced in the earlier time points after birth. This is unsurprising as plenty of evidence indicates that maternal education and smoking are important socio-economic parameters influencing dietary behaviour. In a study by Kiefte de Jong et al., low paternal education, low household income, parental smoking, multiparity, maternal BMI, maternal carbohydrate intake and television watching of the child were determinants of a 'Western-like' diet equivalent to the 'junk food' pattern established in our study $^{(22)}$. However, while Kiefte de Jong et al. primarily focused on toddlers, our study followed study individuals for a significantly longer period of time.

To conclude, this is the first study to identify the dietary patterns in a Central European population during a major socio-economic transition, thereby providing important information regarding the speed and quality of the dietary transition in this population.

\section{Acknowledgements}

Acknowledgements: The authors of this study wish to thank the participating families as well as the gynaecologists, paediatricians, school heads and class teachers who 
took part. Our thanks also go to Dr Lubomír Kukla, PhD, ELSPAC national coordinator 1990-2012, and the entire ELSPAC team. The authors are grateful to Pavel Piler and Vít Kandrnál for valuable comments and cohort description assistance. Financial support: The ELSPAC study is currently supported by the RECETOX Research Infrastructure of Masaryk University CETOCOEN PLUS (CZ.02.1.01/0.0/0.0/15_003/0000469), the National Feasibility Programme of the Ministry of Education of the Czech Republic (LO1214) and by the CELSPAC: Central European Longitudinal Study of Pregnancy and Childhood (MUNI/M/1075/2013) support programme. Conflicts of interest: None. Authorship: M.G. analysed data, drafted and revised the paper. F.Z., L.A. and J.Š. assisted in data analysis. H.P. designed the research, formulated the research question and revised the manuscript draft. J.B.-V. edited and reviewed the manuscript and has primary responsibility for the design of the paper and its final content. Ethics of human subject participation: An ethics committee was established at the international level to support the international legal framework and ethics of the study design. The ELSPAC-CZ study was approved by local ethics committees at the national level. Informed consent was obtained from all study participants during each data collection period. This study was conducted according to the guidelines laid down in the Declaration of Helsinki, and all procedures involving study participants were approved by the local ethics committees at the national level.

\section{Availability of data and materials}

The data that support the findings of this study are available from www.elspac.cz by Research Centre for Toxic Compounds in the Environment (RECETOX) but restrictions apply to the availability of these data, which were used under license for the current study, and so are not publicly available. Data are however available from the authors upon reasonable request and with permission of the Research Centre for Toxic Compounds in the Environment.

\section{Supplementary material}

For supplementary material accompanying this paper visit https://doi.org/10.1017/S1368980020001056

\section{References}

1. Shim J-S, Oh K \& Kim HC (2014) Dietary assessment methods in epidemiologic studies. Epidemiol Health 36, e2014009.

2. Boylan S, Welch A, Pikhart H et al. (2009) Dietary habits in three Central and Eastern European countries: the HAPIEE study. BMC Public Health 9, 439.

3. Bel-Serrat S, Ojeda-Rodríguez A, Heinen MM et al. (2019) Clustering of multiple energy balance-related behaviors in school children and its association with overweight and
Obesity - WHO European Childhood Obesity Surveillance Initiative (COSI 2015-2017). Nutrients 11, 511.

4. Northstone K \& Emmett PM (2008) Are dietary patterns stable throughout early and mid-childhood? A birth cohort study. $\mathrm{Br}$ J Nutr 100, 1069.

5. Northstone K, Smith ADAC, Newby PK et al. (2013) Longitudinal comparisons of dietary patterns derived by cluster analysis in 7- to 13-year-old children. Br J Nutr 109, 2050-2058.

6. Weismayer C, Anderson JG \& Wolk A (2006) Changes in the stability of dietary patterns in a study of middle-aged Swedish women. J Nutr 136, 1582-1587.

7. Mishra GD, McNaughton SA, Bramwell GD et al. (2006) Longitudinal changes in dietary patterns during adult life. Br J Nutr 96, 735-744.

8. Borland SE, Robinson SM, Crozier SR et al. (2008) Stability of dietary patterns in young women over a 2 -year period. Eur J Clin Nutr 62, 119-126.

9. Voráčová J, Sigmund E, Sigmundová D et al. (2015) Changes in eating behaviours among Czech children and adolescents from 2002 to 2014 (HBSC study). Int J Environ Res Public Health 12, 15888-15899.

10. Bienertová-Vašků J, Zlámal F, Pruša T et al. (2017) Parental heights and maternal education as predictors of length/ height of children at birth, age 3 and 19 years, independently on diet: the ELSPAC study. Eur J Clin Nutr 2, 71-85.

11. Koyame-Marsh R (2011) The complexities of economic transition: lessons from the Czech Republic and Slovakia. Int J Bus Soc Sci 2, 71-85.

12. (1989) European Longitudinal Study of Pregnancy and Childhood (ELSPAC). Paediatr Perinat Epidemiol 3, 460-469.

13. Golding J, Pembrey M, Jones R et al. (2001) ALSPAC - the Avon Longitudinal Study of Parents and Children. I. Study methodology. Paediatr Perinat Epidemiol 15, 74-87.

14. Piler P, Kandrnal V, Kukla L et al. (2016) Cohort profile: the European Longitudinal Study of Pregnancy and Childhood (ELSPAC) in the Czech Republic. Int J Epidemiol 46, 1379$1379 f$.

15. CZSO Database of Demographic Indicators for Selected Towns of the Czech Republic | CZSO. Czech Stat. Off. https://www.czso.cz/csu/czso/database-of-demographicindicators-for-selected-towns-of-the-czech-republic (accessed April 2018).

16. Smith ADAC, Emmett PM, Newby PK et al. (2014) Dietary patterns and changes in body composition in children between 9 and 11 years. Food Nutr Res 58, doi: 10.3402/ fnr.v58.22769.

17. Bull CJ \& Northstone K (2016) Childhood dietary patterns and cardiovascular risk factors in adolescence: results from the Avon Longitudinal Study of Parents and Children (ALSPAC) cohort. Public Health Nutr 19, 3369-3377.

18. Northstone K, Smith ADAC, Cribb VL et al. (2014) Dietary patterns in UK adolescents obtained from a dual-source FFQ and their associations with socio-economic position, nutrient intake and modes of eating. Public Health Nutr 17, 1476-1485.

19. Lee S-K \& Sobal J (2003) Socio-economic, dietary, activity, nutrition and body weight transitions in South Korea. Public Health Nutr 6, 665-674.

20. Linando AY \& Lee S-K (2018) Socioeconomic, dietary, nutrition, body weight and epidemiologic transitions: three nations at different stage of development. Prog Nutr 20, 602-615.

21. MacKeown JM, Cleaton-Jones PE, Edwards AW et al. (1998) Energy, macro- and micronutrient intake of 5-year-old urban black South African children in 1984 and 1995. Paediatr Perinat Epidemiol 12, 297-312.

22. Kiefte-de Jong JC, de Vries JH, Bleeker SE et al. (2013) Sociodemographic and lifestyle determinants of 'Western-like' and 'Health conscious' dietary patterns in toddlers. BrJ Nutr $\mathbf{1 0 9}$, 137-147. 